\title{
The Long-Term Impact of Early Life Poverty on Orbitofrontal Cortex Volume in Adulthood: Results from a Prospective Study Over 25 Years
}

\author{
Nathalie E Holz', Regina Boecker', Erika Hohm', Katrin Zohsel', Arlette F Buchmann', \\ Dorothea Blomeyer', Christine Jennen-Steinmetz ${ }^{2}$, Sarah Baumeister', Sarah Hohmann', \\ Isabella Wolf ${ }^{1,3}$, Michael M Plichta ${ }^{4}$, Günter Esser ${ }^{5}$, Martin Schmidt', Andreas Meyer-Lindenberg ${ }^{4}$, \\ Tobias Banaschewski', Daniel Brandeis ${ }^{1,6,7}$ and Manfred Laucht*, 1,5,7 \\ 'Department of Child and Adolescent Psychiatry and Psychotherapy, Central Institute of Mental Health, Medical Faculty Mannheim/Heidelberg \\ University, Mannheim, Germany; ${ }^{2}$ Department of Biostatistics, Central Institute of Mental Health, Medical Faculty Mannheim/Heidelberg \\ University, Mannheim, Germany; ${ }^{3}$ Department of Neuroimaging, Central Institute of Mental Health, Medical Faculty Mannheim/Heidelberg \\ University, Mannheim, Germany; ${ }^{4}$ Department of Psychiatry and Psychotherapy, Central Institute of Mental Health, Medical Faculty Mannheim/ \\ Heidelberg University, Mannheim, Germany; ${ }^{5}$ Department of Psychology, University of Potsdam, Potsdam OT Golm, Germany; ${ }^{6}$ Department of \\ Child and Adolescent Psychiatry, University of Zurich, Zurich, Switzerland
}

\begin{abstract}
Converging evidence has highlighted the association between poverty and conduct disorder (CD) without specifying neurobiological pathways. Neuroimaging research has emphasized structural and functional alterations in the orbitofrontal cortex (OFC) as one key mechanism underlying this disorder. The present study aimed to clarify the long-term influence of early poverty on OFC volume and its association with CD symptoms in healthy participants of an epidemiological cohort study followed since birth. At age 25 years, voxelbased morphometry was applied to study brain volume differences. Poverty $(0=$ non-exposed $(N=134), I=\operatorname{exposed}(N=33))$ and smoking during pregnancy were determined using a standardized parent interview, and information on maternal responsiveness was derived from videotaped mother-infant interactions at the age of 3 months. CD symptoms were assessed by diagnostic interview from 8 to 19 years of age. Information on life stress was acquired at each assessment and childhood maltreatment was measured using retrospective self-report at the age of 23 years. Analyses were adjusted for sex, parental psychopathology and delinquency, obstetric adversity, parental education, and current poverty. Individuals exposed to early life poverty exhibited a lower OFC volume. Moreover, we replicated previous findings of increased CD symptoms as a consequence of childhood poverty. This effect proved statistically mediated by OFC volume and exposure to life stress and smoking during pregnancy, but not by childhood maltreatment and maternal responsiveness. These findings underline the importance of studying the impact of early life adversity on brain alterations and highlight the need for programs to decrease income-related disparities.
\end{abstract}

Neuropsychopharmacology (20I5) 40, 996-I004; doi:I0.I038/npp.20I4.277; published online I0 December 20I4

\section{INTRODUCTION}

About one-sixth of the population in Western countries has been reported to be at risk of poverty (Destatis, 2013; Short, 2013). Consistent evidence indicates that poverty is a main determinant of increased psychopathology such as conduct disorder (CD) (Costello et al, 2003; Evans et al, 2013). CD is characterized by a persistent pattern of rule violation and antisocial behavior including aggressive, deceptive, and

* Correspondence: Dr M Laucht, Department of Child and Adolescent Psychiatry and Psychotherapy, Central Institute of Mental Health, Medical Faculty Mannheim/Heidelberg University, J5, Mannheim 68I59, Germany, Tel: +49 62I 1703 4903, Fax: +49 62। 1703 1205,

E-mail: manfred.laucht@zi-mannheim.de

${ }^{7}$ The last two authors contributed equally to this work.

Received 16 July 2014; revised 24 September 2014; accepted 10 October 20।4; accepted article preview online 15 October 2014 destructive behaviors toward peers and adults (American Psychiatric Association, 1994). In their natural experiment including income intervention, Costello et al (2003) demonstrated that poverty is linked to the development of $\mathrm{CD}$ implying social causation pathways, that is, exposure to adversity and stress. Numerous studies have confirmed the relationship between poverty and externalizing psychopathology (eg, Emerson et al, 2011; Midouhas et al, 2013). Moreover, the orbitofrontal cortex (OFC) was identified as one region that is compromised in $\mathrm{CD}$ and antisocial behavior (Blair, 2004; Rubia, 2011). This region is typically involved during the regulation of emotional or motivational behavior (Rubia, 2011). Consistent with this, a number of studies have found blunted neural activity during reward processing (Rubia et al, 2009) and passive avoidance learning (Finger et al, 2011), indicating deficient learning of reward or punishment contingencies, which may, in turn, 
result in less effective decision making. Likewise, a decreased OFC volume has been found in CD patients (Huebner et al, 2008; Yang and Raine, 2009; Fairchild et al, 2011).

Given these relationships of both poverty and OFC deficits with $\mathrm{CD}$, an intriguing question is whether poverty increases the risk for $\mathrm{CD}$ by affecting OFC integrity. However, this hypothesis has not been tested in previous work, and only a small number of neuroimaging studies have investigated the impact of childhood poverty/low socioeconomic status (SES, including poverty and low parental education) on brain structure and function even in a cross-sectional design, with longitudinal data in adults being rare. In children, the majority of studies have revealed deficits in various brain regions. As an example, Luby et al (2013) found smaller amygdala and hippocampal volumes in poor children, with the latter being mediated by caregiver support. In contrast, poverty has been linked to smaller hippocampal, but unaltered amygdala volumes in children and adolescents (Hanson et al, 2011; Noble et al, 2012), whereas no such associations were observed by Butterworth et al (2012). Furthermore, in infants from a poor family background, lower frontal and parietal gray matter volumes have been reported, which were found to be associated with externalizing psychopathology (Hanson et al, 2013). Likewise, SES has been related to cortical thickness in the anterior cingulate gyrus and the left superior frontal gyrus (Lawson et al, 2013). However, this relationship appeared to be mainly driven by parental education. Finally, Jednorog et al (2012) demonstrated an effect of SES on the volumes of various brain regions, including the insula, superior frontal gyrus, and hippocampus. On a functional level, poverty has been related to less prefrontal activity during emotion regulation, which was found to be mediated by chronic stress exposure (Kim et al, 2013) and less default-mode network connectivity, which was inversely related to stress reactivity (Sripada et al, 2014). Although previous research has provided preliminary evidence of structural alterations in children exposed to poverty, no study so far has examined the long-term impact of this early life adversity on brain anatomy until adulthood.

Likewise, given the cross-sectional nature of previous studies (eg, Noble et al, 2012), control for additional confounds may not have been possible. As an example, poverty and poor parental education are known to be closely linked, although they might have differential effects on health outcomes (Lawson et al, 2013). Moreover, low SES has been associated with poor birth outcomes such as low birth weight and preterm birth (Potijk et al, 2013). Likewise, history of parental psychopathology (Shelton et al, 2011) should be considered to exclude a shared genetic liability. Finally, given the fact that poverty is a distal environmental risk factor, more proximal measures such as stressful life events (SLE) are likely to mediate its impact (Kim et al, 2013). Moreover, additional pathways have to be considered when investigating the specific effect of poverty on externalizing psychopathology and brain morphology such as exposure to SLEs (Flouri et al, 2013), smoking during pregnancy (Holz et al, 2014), childhood maltreatment (Kunitz et al, 1998), and maternal support (Luby et al, 2013), all of which being increased in poor families (Eiden et al, 2013; Kim et al, 2013; Eckenrode et al, 2014).
Furthermore, $\mathrm{CD}$ is highly comorbid with ADHD (Hamshere et al, 2013) and substance abuse (Carpentier et al, 2012), which also have to be taken into account.

Using longitudinal data from an epidemiological cohort study of 167 young adults with no current psychopathology followed since birth (Laucht et al, 2000), the present investigation allowed us to account for all the confounders previously mentioned when assessing the specific long-term effect of early life poverty on OFC volume during adulthood. Furthermore, we aimed to (i) replicate the well-established link between poverty and child and adolescent CD, (ii) examine the association of OFC volume alterations with $\mathrm{CD}$, and (iii) test for a possible mediation of the impact of poverty on CD by SLEs, smoking during pregnancy, childhood maltreatment, and maternal responsiveness, as well as with current OFC volume, which is assumed to be a stable trait-like marker (Blair, 2004).

\section{MATERIALS AND METHODS}

\section{Sample}

This investigation was conducted in the framework of the Mannheim Study of Children at Risk, an ongoing epidemiological cohort study of the long-term outcome of early risk factors (for full details cf., Laucht et al, 2000 and see Supplementary Materials and Methods). Assessments were conducted at the age of 3 months and at regular intervals throughout development, most recently at the age of 25 years (Supplementary Figure 1). Psychopathology was assessed by the Structured Clinical Interview for DSM-IV (SCID-I German version; Wittchen et al, 1997), and only healthy participants were included. The study was approved by the ethics committee of the University of Heidelberg and written informed consent was obtained from all participants.

\section{Assessments}

Early life Poverty. Early life poverty was determined by a standardized interview conducted with the mother at the 3-month assessment and defined as an income level below the risk-of-poverty threshold, which is set at $60 \%$ of the national median equivalized disposable income adjusted for household size according to the Federal Statistical Office of Germany (Destatis, 2012), resulting in an dichotomous variable $(0=$ not exposed and $1=$ exposed $)$. This definition of poverty is also commonly used in international contexts (Emerson et al, 2011; Lelkes and Gasior, 2011; Destatis, 2012). From $N=167$ participants, 33 (19.8\%) were categorized as being at risk of poverty (details in Supplementary Materials and Methods). In addition, we validated our results using the log-transformed income-to-needs ratio (US Census Bureau, 2004), to account for the nonlinearity and to weight the effect of being at risk of poverty more than the differentiation in the upper income range (respective methods and results are described in the supplement).

Covariates and Confounders. The results were adjusted for several important confounders, such as sex, parental characteristics (education, psychopathology, and delinquency), obstetric adversity, and current poverty (see 
Supplementary Materials and Methods for details). To address confounding with regard to $\mathrm{CD}$, we, additionally, assessed lifetime ADHD symptoms and lifetime substance abuse, including nicotine, alcohol, and cannabis abuse (see Supplementary Materials and Methods).

Life Stress. To assess exposure to life stress (LS), a semistructured parent interview was conducted at each assessment until the age of 15 years. From the age of 19 years onwards, the young adults were interviewed. The interview, a modified and shortened version of the Munich Events List (Maier-Diewald et al, 1983), evaluated the occurrence of adverse life events during a period of 1 year before the assessment. The items (42-59, adjusted for developmental period) covered all relevant areas of children's and young adults' LS, including family, school, parents, health, legal troubles, and living conditions, such as birth of a sibling, death of a close relative, or parents' separation. Two composite scores were computed by summing up the $z$-standardized scores, depending on the time point of the outcome assessment (details in Supplementary Materials and Methods).

Prenatal Maternal Smoking. Maternal smoking during pregnancy was determined by a standardized interview with the mother conducted at the 3-month assessment. In all, $77.2 \%(N=129)$ mothers were nonsmokers, $8.4 \%$ $(N=14)$ reported smoking 1-5 cigarettes per day, and $14.4 \%(N=24)$ more than 5 cigarettes per day as previously described by Holz et al (2014).

Maternal Responsiveness. An observational procedure was used to assess the quality of mother-infant interaction at the participant's age of 3 months as described by Laucht et al (2001). Mother-infant dyads were observed and videotaped during a 10-min semistructured session at our laboratory. A score for maternal responsiveness in interaction with her infant was computed by summing up the mother scales, including emotion, physical affect, vocalization, lack of verbal restrictions, congruency, variability, reactivity, and stimulation, across minutes (mean $=317.43$, $\mathrm{SD}=34.80$ ). More detailed information can be found in the supplement.

Childhood Maltreatment. At the age of 23 years, participants completed the brief screening version of the Childhood Trauma Questionnaire (CTQ; Bernstein et al, 2003). The CTQ entails a retrospective assessment of five types of self-reported childhood maltreatment, that is, sexual, physical, and emotional abuse, as well as emotional and physical neglect (details in Supplementary Materials and Methods). The CTQ yields a total score of each type of maltreatment, which was used in the current investigation.

CD Symptoms. CD symptoms according to DSM-IV were assessed in 165 individuals using the Mannheim Parent Interview (Esser et al, 1989) at the age of 8 and 11 years and the Schedule for Affective Disorders and Schizophrenia for School-Age Children (Kaufman et al, 1996) at the age of 15 and 19 years. The MEI is a highly structured interview adapted from Rutter's parent interviews, which was modified to include all symptoms related to major DSMIV diagnoses, and has been shown to be a sensitive measure of child disturbance (Holz et al, 2014). The K-SADS is a widely used structured diagnostic interview completed independently with parents and children (at the age of 19 years with adolescents only). A sum score of the $z$-transformed values was computed. Further details on psychometric properties are in Supplementary Materials and Methods. Supplementary Table S1 lists the number of subjects with DSM-IV CD diagnosis (American Psychiatric Association, 1994), the rates being well in line with the known higher prevalence in boys compared with girls during childhood and adolescence (Nock et al, 2006). None of them was in the clinical range at age 25 years.

Voxel-Based Morphometry. We acquired $1 \times 1 \times 1 \mathrm{~mm}^{3}$ T1-weighted anatomical images with 192 slices covering the whole brain (matrix $256 \times 256$, repetition time $=2300 \mathrm{~ms}$, echo time $=3.03 \mathrm{~ms}, 50 \%$ distance factor, field of view $256 \times 256 \times 192 \mathrm{~mm}^{3}$, flip angle $9^{\circ}$ ) using a $3 \mathrm{~T}$ scanner (Magnetom TRIO; Siemens, Erlangen, Germany) with a standard 12-channel head coil. Images were bias-corrected and classified into gray matter, white matter, cerebrospinal fluid, and non-brain tissue using Diffeomorphic Anatomical Registration through Exponentiated Lie algebra registration toolbox (Ashburner, 2007). Data were segmented based on tissue probability maps, indexing the prior probability of any voxel in a registered image being grey or white matter, or cerebrospinal fluid. An average template from the data was created, to which the images were registered. Additionally, the images were affine-transformed to MNI space and smoothed with an 8-mm FWHM kernel. Total intracranial volume was calculated by adding the tissue probabilities of gray matter, white matter, and cerebrospinal fluid and used as a covariate. For group statistics of gray matter images, early life poverty $(0=$ not present and $1=$ present) was entered as a covariate of interest while additionally controlling for the covariates previously mentioned. To test our a priori hypothesis, as described in previous research (Blair, 2004; Rubia, 2011; Blair, 2013), anatomical ROI masks comprising the OFC were derived from the WFU PickAtlas v.2.4 (Maldjian et al, 2003), where family-wise error (FWE) correction was applied with a threshold of $p<0.05$. Additionally, exploratory whole brain analyses were performed with a $p<0.001$ uncorrected threshold and a criterion of 10 adjacent voxels. For display purposes, the statistical threshold was set at $p=0.005$ uncorrected in the ROI. To visualize and further analyze the effects using regression analysis, mean contrast values of each participant were extracted from the significant cluster and exported to SPSS Statistics 20 (IBM, Armonk, NY). Given the previously reported association of the OFC with CD (Blair, 2004; Rubia, 2011), we examined whether brain volume was related to $\mathrm{CD}$ symptoms, performing partial correlations controlled for the covariates previously mentioned.

Mediation. Mediation was tested under consideration of all covariates previously mentioned (see Supplementary Materials and Methods). 
Table I Sample Characteristics

\begin{tabular}{|c|c|c|c|}
\hline Early life poverty & Non-exposed & Exposed & p-Value \\
\hline$N(\%)$ & $134(80.2)$ & $33(19.8)$ & \\
\hline Males, N (\%) & $54(40.3)$ & $13(39.39)$ & $0.93^{\mathrm{a}}$ \\
\hline Parental psychiatric disorder, N (\%) & $16(11.94)$ & $9(27.27)$ & $0.03^{\mathrm{a}}$ \\
\hline Parental delinquency, N (\%) & I। (8.20) & $7(21.21)$ & $0.03^{\mathrm{a}}$ \\
\hline CD symptoms, M (SD) & $-0.4 \mathrm{I}(0.90)$ & $0.19(1.74)$ & $0.03^{c}$ \\
\hline ADHD symptoms, M (SD) & $1.64(2.53)$ & $3.39(3.21)$ & $0.006^{d}$ \\
\hline Lifetime nicotine dependence, M (SD) & $-0.22(3.42)$ & $0.56(3.62)$ & $0.25^{\mathrm{b}}$ \\
\hline Lifetime alcohol abuse, M (SD) & $-0.69(2.39)$ & $-0.39(2.78)$ & $0.54^{\mathrm{b}}$ \\
\hline Lifetime cannabis use, M (SD) & $0.46(1.04)$ & $0.70(1.02)$ & $0.25^{b}$ \\
\hline Smoking during pregnancy, M (SD) & $0.24(0.56)$ & $0.91(1.01)$ & $<0.001^{\mathrm{e}}$ \\
\hline CTQ score, M (SD) & $29.5(6.11)$ & $30.25(5.74)$ & $0.53^{\mathrm{e}}$ \\
\hline Maternal responsiveness, M (SD) & $320.3(30.22)$ & $305.76(48.07)$ & $0.04^{e}$ \\
\hline
\end{tabular}

Abbreviations: M, mean; SD, standard deviation.

${ }^{a} \chi^{2}$ test.

$b_{t-\text { Test }}(\mathrm{df}=165)$.

${ }^{c} N=165$, adjusted for sex, parental characteristics (education, psychopathology, and delinquency), and obstetric adversity.

${ }_{t}$-Test $(\mathrm{df}=162)$.

eAdjusted for sex, parental characteristics (education, psychopathology, and delinquency), and obstetric adversity.

\section{RESULTS}

\section{Sample Characteristics}

As expected, higher rates of parental psychiatric disorder and delinquency were found in the group at risk of early poverty compared with those non-exposed. Moreover, in line with previous research, the poverty group exhibited more $\mathrm{CD}$ and $\mathrm{ADHD}$ symptoms and were exposed to higher levels of LS and smoking during pregnancy, as well as to less maternal responsiveness. No group differences with regard to gender, obstetric adversity, parental education, current poverty, total intracranial volume, lifetime substance abuse, and CTQ emerged (Table 1).

In contrast, the association between the log-transformed income-to-needs ratio and $\mathrm{CD}$ just failed to reach significance ( $p=0.05$; see Supplementary Results).

\section{Voxel-Based Morphometry}

Individuals exposed to early life poverty $(N=33)$ exhibited a smaller OFC volume, when adjusted for total intracranial volume, sex, parental characteristics (education, psychopathology, and delinquency), obstetric adversity, and current poverty, when compared with those not exposed to poverty $\left(N=134 ; t(158)=3.80, p_{\mathrm{FWE}}=0.02\right.$, peak 6 , 45-18; Figure 1). Further volume decreases in those exposed to early poverty when compared with the non-exposed group were found in the inferior temporal gyrus, superior orbitofrontal gyrus, middle temporal gyrus, precentral gyrus, medial frontal gyrus, and the bilateral insula using a more liberal threshold of $p_{\text {uncorr }}=0.001$. There was no region showing volume increases in individuals exposed to poverty. After adjustment for $\mathrm{CD}$ symptoms as an additional covariate, the results remained significant $(t(155)=$ $3.46, p_{\mathrm{FWE}}=0.045$ ). In contrast to early poverty, current poverty was unrelated to differences in OFC volume $\left(t(157)=2.08, p_{\mathrm{FWE}}=0.80\right)$. When using the continuous log-transformed income-to-needs ratio as a covariate of interest, the results were even more significant (described in Supplementary Results).

\section{Association Between OFC Volume and CD}

There was an inverse association between OFC volume and CD symptoms $(r=-0.19, p=0.01$; Figure 2$)$, indicating that $\mathrm{OFC}$ volume decreased with the number of $\mathrm{CD}$ symptoms.

\section{Mediation}

A significant effect of exposure to early life poverty on CD symptoms was obtained $(\beta=0.99, \mathrm{SE}=0.476, p=0.039$; Figure 3a). Including SLEs as a mediator revealed a significant indirect effect (Sobel test, $p=0.037$ ), with a significant path between exposure to poverty and LS ( $\beta=1.59$, $\mathrm{SE}=0.70, p=0.025)$, and between LS and CD symptoms $(\beta=0.27, \mathrm{SE}=0.05, p<0.001)$. Moreover, the direct path between poverty and $\mathrm{CD}$ was no longer significant $(\beta=0.58$, 

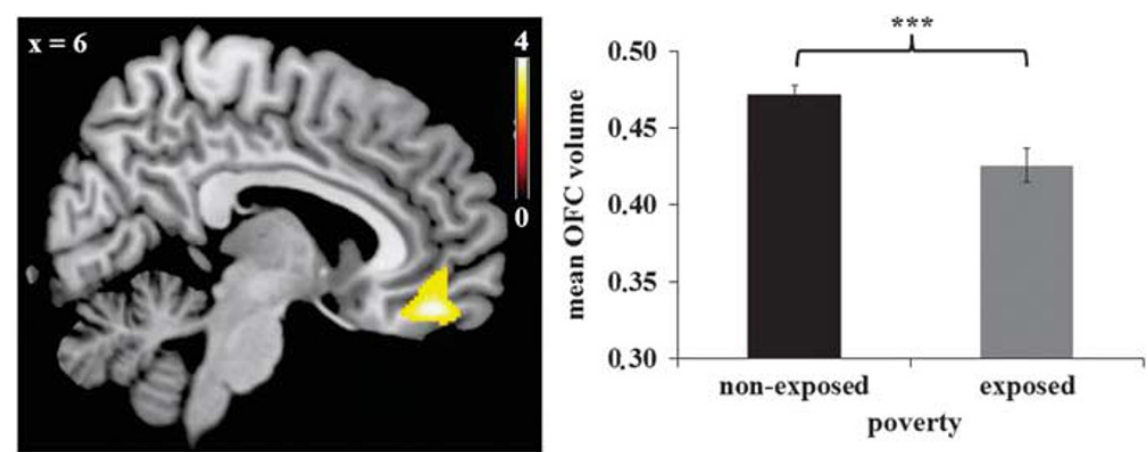

Figure I Effect of exposure to early life poverty on orbitofrontal cortex (OFC) volume. Left: Volume is decreased in individuals exposed to poverty $\left(P_{F W E}=0.02\right)$. Right: Corresponding parameter estimates of the non-exposed and the exposed groups $(N=167$; mean $\pm S E M$ are indicated, $* * * * 2<0.00 I)$.

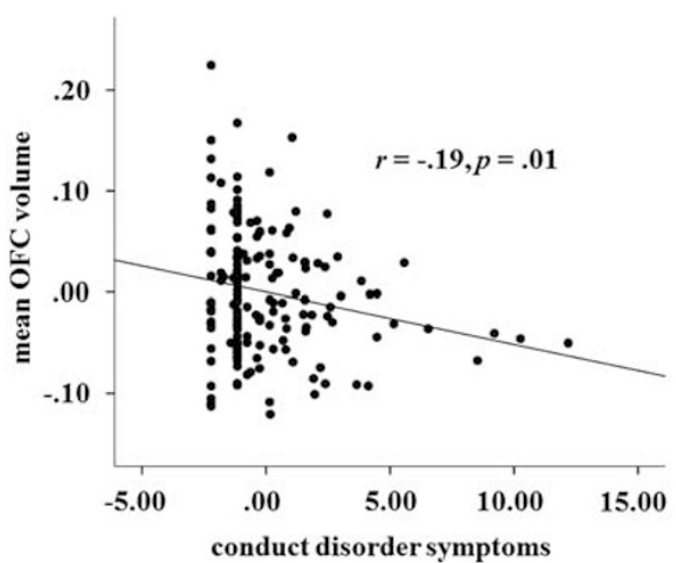

Figure 2 Scatter plot of the correlation between the significant cluster in the orbitofrontal cortex (OFC) and conduct disorder (CD) symptoms $(N=165)$

$\mathrm{SE}=0.45, p=0.20$ ), suggesting that the effect was fully mediated by LS. In contrast, OFC volume proved to be not mediated by LS (Sobel test $p=0.97$ ), with no significant path between LS and OFC volume $(\beta=0.00, \mathrm{SE}=0.001$, $p=0.82)$. Accordingly, the effect of poverty on OFC volume remained significant $(\beta=-0.05, \mathrm{SE}=0.01, p<0.001)$. In addition, smoking during pregnancy mediated the relationship between poverty and CD ( $p=0.004$; Figure $3 \mathrm{~b})$, with significant paths between poverty and smoking during pregnancy $(\beta=0.57, \mathrm{SE}=0.13, p<0.001)$ and between smoking during pregnancy and $\mathrm{CD}(\beta=1.05, \mathrm{SE}=0.29$, $p<0.001)$. The effect of poverty on CD was no longer significant $(\beta=0.40, \mathrm{SE}=0.49, p=0.82)$, indicating full mediation. However, mediation did not apply to the association between poverty and OFC volume $(p=0.10)$, which remained significant when including smoking during pregnancy $\left(t(156)=3.33, p_{\mathrm{FWE}}=0.04\right)$. Thus, in addition to life events, we present another possible pathway by which poverty may exert its effects on CD. In contrast, maternal responsiveness and childhood trauma did neither mediate the association of poverty with $\mathrm{CD}$ nor with OFC volume (all $p>0.92$ ). Finally, we were interested in whether the effect of poverty on CD symptoms could have been mediated by OFC volume decrease. The Sobel test revealed full mediation ( $p=0.04$; Figure $3 c$ ), comprising significant paths between poverty and OFC volume $(\beta=-0.05$,
$\mathrm{SE}=0.01, p<0.001)$ and OFC volume and CD $(\beta=-8.41$, $\mathrm{SE}=3.44, p=0.02)$, with the direct effect being no longer significant $(\beta=0.61, \mathrm{SE}=0.49, p=0.22)$.

\section{Role of Comorbidity}

Considering the impact of comorbid psychopathology, our results suggest that substance abuse neither had a significant impact on the association between poverty and OFC volume $\left(t(155)=3.7, p_{\mathrm{FWE}}=0.02\right)$ nor on the association between poverty and $\mathrm{CD}(\beta=0.945, \mathrm{SE}=0.46, p=0.04)$. No significant partial correlations between OFC volume and the different substance abuse scores were found (all $p>0.26$ ). In contrast, consistent with the well-known comorbidity between $\mathrm{CD}$ and $\mathrm{ADHD}$, the association between poverty and $C D$ remained no longer significant when additionally controlled for ADHD $(\beta=0.209, \mathrm{SE}=0.40, p=0.61)$. However, the association with OFC volume was unaffected $\left(t(154)=3.51, p_{\mathrm{FWE}}=0.04\right)$. Likewise, under consideration of all covariates, lifetime $\mathrm{ADHD}$ symptoms showed an inverse relationship with OFC volume at a trend level $(r=-0.15, p=0.06)$.

\section{DISCUSSION}

The investigation reported here provides first evidence of the long-term impact of early life poverty on OFC volume in young adults. Specifically, our results from a prospective study over 25 years indicate that individuals who were born into poor circumstances exhibited a decreased volume in the OFC, irrespective of a range of potential confounders. Moreover, we replicated pertinent findings of increased $\mathrm{CD}$ symptoms as a consequence of childhood poverty, with this effect being mediated by the exposure to LS, smoking during pregnancy, and OFC volume decrease.

The OFC is referred to as a hot paralimbic region (Rubia, 2011), which is particularly involved in the top-down regulation of emotional and motivational behavior, impulse control, as well as goal-directed behavior (Davidson et al, 2000). OFC activation is typically elicited during the perception of angry faces (Blair, 2004) as well as during the processing of rewards, which are both crucial for social behavior (Kringelbach and Rolls, 2004). Remarkably, this structure has previously been found to be associated with 
a

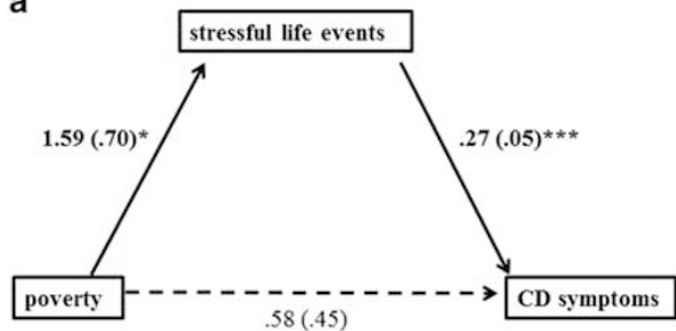

C

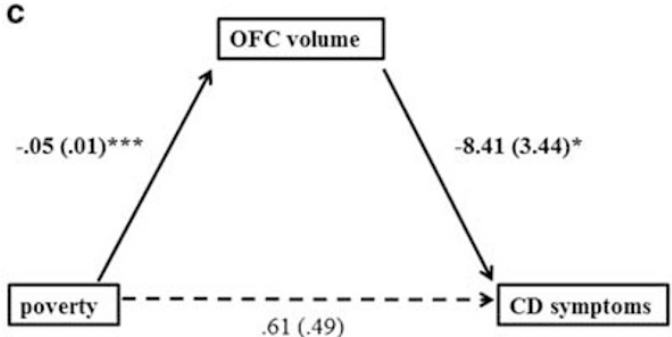

b

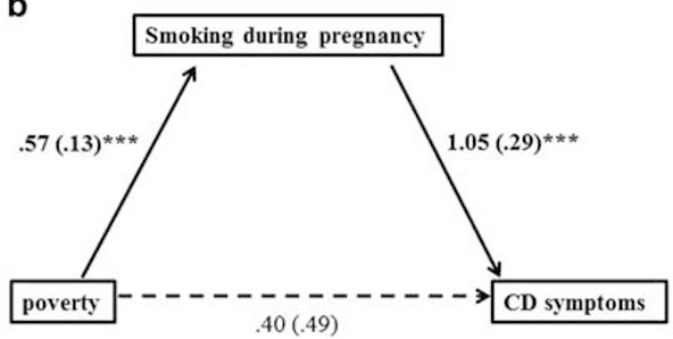

Figure 3 Mediation analysis. Path model showing that life stress (a, from age 3 months up to 19 years, $p=0.04$ ), smoking during pregnancy ( $b, p=0.004$ ) and orbitofrontal cortex $(\mathrm{OFC})$ volume $(c, p=0.04)$ fully mediated the association between exposure to poverty and conduct disorder $(C D)$ symptoms.

environmental risk (eg, Meyer-Lindenberg and Tost, 2012). In addition, a decreased OFC response has been shown to predict aggressive behavior (Coccaro et al, 2007), whereas reduced volumes have been observed in violent, antisocial, and psychopathic individuals (Yang and Raine, 2009). CD is characterized by aggression, destructive behavior, deceitfulness or theft, and the violation of rules, suggesting a key regulatory deficit. A smaller volume in the OFC might lead to impairment in integrating emotional and motivational features of social interactions, which, in turn, might result in an inability to reflect the consequences of actions and subsequently give rise to antisocial and violent behavior. In line with this suggestion, we found that a smaller OFC volume was related to more conduct problems.

As poverty does not exert its influence on the individual in a direct manner, more proximal measures are likely to mediate the effect. In general, three possible pathways may link poverty and health. First, psychosocial adversity (Luby et al, 2013), such as exposure to LS, could be considered as a possible mediator of the impact of poverty on behavioral or brain outcomes. Although our findings provided support for this hypothesis with regard to CD symptoms, no such mediation was found with regard to OFC volume. In contrast, childhood maltreatment and maternal responsiveness were found to not impact the relationship of poverty with $\mathrm{CD}$ and $\mathrm{OFC}$ volume. In addition to a mediating role of LS, we found smoking during pregnancy as mediating the relationship between poverty and $\mathrm{CD}$. This is well in line with studies revealing increased rates of smoking during pregnancy in women from a low-income background (Eiden et al, 2013), suggesting a second pathway, that is, increased exposure to substances during pregnancy, by which poverty may exerts its influence. A third material perspective that has been discussed, which could not be examined here, highlights that living in poverty results in diminished access to resources and to malnutrition (Lynch et al, 2000). For example, vitamin D deficiency leads to abnormal brain development such as reduced neuronal differentiation, with vitamin $\mathrm{D}$ receptors being prevalent in the orbital and cingulate cortices (Eyles et al, 2009). Notably, vitamin D intake is lower in people living in poverty (Moore et al, 2014). Most likely, these pathways are not mutually exclusive and may rather act in concert to promote the indirect effect of poverty on health.

Interestingly, when considering the role of comorbidity, ADHD but not substance abuse significantly impacted the relationship between poverty and CD. This is not surprising with regard to studies showing that about $30-50 \%$ of children exhibit comorbid CD and ADHD (Hamshere et al, 2013). However, conduct problems often precede the development of substance abuse (Hawkins et al, 1992), which may explain why the association between poverty and CD remained significant under consideration of substance abuse. In contrast, none of the comorbid symptoms had an impact on the association with OFC volume, which may speak for a higher sensitivity of the brain outcome. Consistent with the role of the OFC in emotional and motivational behavior (Davidson et al, 2000) and aggression (Coccaro et al, 2007), an inverse relationship of OFC volume was found with CD but not with ADHD or substance abuse.

Several limitations of our study have to be considered when evaluating the results. First, CD symptoms were not assessed concurrently with structural MRI data; thus, cause-effect relationships are difficult to establish. Although the latter were collected during young adulthood (at the age of 25 years), CD symptoms were obtained during childhood and adolescence (between the ages of 8 and 19 years), a developmental period characterized by a high prevalence of antisocial behavior. Moreover, as the MRI findings were obtained in currently healthy individuals, they may be interpreted as indicating a higher susceptibility to $\mathrm{CD}$ or even the long-term vestiges of this disorder into adulthood. However, our MRI results could not be attributed to $\mathrm{CD}$, as adjustment for $\mathrm{CD}$ symptoms did not 
change the principal finding. In accordance with previous reports, suggesting that the OFC functions as a stable marker and mediator of aggressive behavior (Blair, 2004), we found that the relationship between poverty and CD symptoms was mediated by OFC volume decrease. However, this finding has to be interpreted with caution, given the temporal order constraints of conventional mediation analysis. Thus, future studies should assess both outcomes simultaneously to confirm this finding. In this line, although both smoking during pregnancy and early life poverty were assessed at the same time point (at the age of 3 months), we assumed, based on the pertinent literature, that poverty preceded pregnancy (Eiden et al, 2013). However, we cannot be sure whether smoking during pregnancy per $s e$ is the mediating agent or possible neurobiological consequences acting in early life, such as negative effects of nicotine on acetylcholine receptors and cholinergic neurotransmission in the offspring (eg, Slotkin et al, 2004). Second, only a small proportion of our sample was at risk of poverty. Although this rate is even above the general rates of poverty in Western countries (Destatis, 2013; Short, 2013), it may result in an underpowered analysis and could have biased the findings. Therefore, we validated our MRI finding using the income-to-needs ratio as a continuous measure of poverty. Although the impact on OFC volume was robustly confirmed and was even more significant, the effect on CD symptoms fell just short of significance $(p=0.05)$. This may imply that both effects are promoted via different neural trajectories, comprising, on the one hand, a pure linear, and, on the other hand, a nonlinear relationship. In contrast to the impact on OFC volume, the effect on $C D$ may rather reflect a general impact of being at risk of poverty, including, among others, inadequate conditions of health care and nutrition, as well as the self-perception of being underprivileged. It is likely that there is no linear association in the upper range of the income-to-needs ratio, that is, rich people are not more prone to develop CD when compared with richer ones indicating that effects are likely to arise in the lower range of poverty (Barajas et al, 2008). In line with this, previous studies have provided evidence for a robust categorical relationship between poverty and CD (eg, Costello et al, 2003; Emerson et al, 2011; Midouhas et al, 2013). Third, genetic aspects may have a role in mediating the effect of poverty on OFC volume. However, by controlling for parental mental health and delinquency, we partly accounted for a possible genetic impact. Fourth, poverty is a distal measure associated with a variety of variables, which may be society-specific, that is, poverty may imply different confounding factors in different societies. Thus, any association with health outcomes may not be generalizable across societies. Fifth, we do not have data on maternal dietary patterns during pregnancy. However, poverty is a summary of different mechanisms converging and thus provides a strong measure of cumulating risks. Future studies are warranted to clarify the role of diet.

In conclusion, poverty may have a deleterious long-term impact on OFC structure, with lower volumes being related to $\mathrm{CD}$. In this respect, the present study contributes to providing a better understanding of the mechanisms underlying the detrimental effects of early environmental adversity on brain development and behavior during later life. Hence, our results strengthen the importance of early postnatal intervention programs aiming to minimize income-related disparities.

\section{FUNDING AND DISCLOSURE}

This work was supported by grants from the German Research Foundation (grant number DFG LA 733/1-2) to ML, DB, AML, and TB. The funding source had no role in study design, in collection, analysis, and interpretation of data, in the writing of the report, and in the decision to submit the article for publication. TB served in an advisory or consultancy role for Hexal Pharma, Lilly, Medice, Novartis, Otsuka, Oxford outcomes, PCM Scientific, Shire, and Viforpharma. He received conference attendance support and conference support or received speaker's fee by Lilly, Medice, Novartis, and Shire. He is/has been involved in clinical trials conducted by Lilly, Shire, and Viforpharma. AML receives consultant fees and travel expenses from AstraZeneca, Hoffmann-La Roche, Lundbeck Foundation, speaker's fees from Pfizer Pharma, Lilly Deutschland, Glaxo SmithKline, Janssen Cilag, BristolMyers Squibb, Lundbeck, and AstraZeneca.

\section{ACKNOWLEDGEMENTS}

We thank Sibylle Heinzel, Elisabeth Reichert, Anna Becker, Angelika Bocklage, Andrea Len, Daniel Megally, and Elise Jezycki for conducting and supporting the assessments.

\section{REFERENCES}

American Psychiatric Association (1994). Diagnostic and Statistical Manual of Mental Disorders. 4th edn. American Psychiatric Association: Washington, DC.

Ashburner J (2007). A fast diffeomorphic image registration algorithm. NeuroImage 38: 95-113.

Barajas RG, Philipsen N, Brooks-Gunn J (2008). Cognitive and emotional outcomes for children in poverty. In: Crane DR, Heaton $\mathrm{T}$ (eds). Handbook of Families and Poverty. Sage Publications: New York, NY, pp 311-333.

Bernstein DP, Stein JA, Newcomb MD, Walker E, Pogge D, Ahluvalia T et al (2003). Development and validation of a brief screening version of the Childhood Trauma Questionnaire. Child Abuse Negl 27: 169-190.

Blair RJ (2004). The roles of orbital frontal cortex in the modulation of antisocial behavior. Brain Cogn 55: 198-208.

Blair RJ (2013). The neurobiology of psychopathic traits in youths. Nat Rev Neurosci 14: 786-799.

Butterworth P, Cherbuin N, Sachdev P, Anstey KJ (2012). The association between financial hardship and amygdala and hippocampal volumes: results from the PATH through life project. Soc Cogn Affect Neurosci 7: 548-556.

Carpentier PJ, Knapen LJ, van Gogh MT, Buitelaar JK, De Jong CA (2012). Addiction in developmental perspective: influence of conduct disorder severity, subtype, and attention-deficit hyperactivity disorder on problem severity and comorbidity in adults with opioid dependence. J Addict Dis 31: 45-59.

Coccaro EF, McCloskey MS, Fitzgerald DA, Phan KL (2007). Amygdala and orbitofrontal reactivity to social threat in individuals with impulsive aggression. Biol Psychiatry 62: 168-178. 
Costello EJ, Compton SN, Keeler G, Angold A (2003). Relationships between poverty and psychopathology: a natural experiment. JAMA 290: 2023-2029.

Davidson RJ, Putnam KM, Larson CL (2000). Dysfunction in the neural circuitry of emotion regulation-a possible prelude to violence. Science 289: 591-594.

Destatis (2012). Wirtschaftsrechnungen-Einkommens- und Verbrauchsstichprobe-Einkommensverteilung in Deutschland, Fachserie 15, Heft 6. Statistisches Bundesamt. Available at: https://www.destatis.de/DE/Publikationen/Thematisch/Einkommen KonsumLebensbedingungen/EinkommenVerbrauch/Einkommens verteilung2152606089004.pdf?_blob=publicationFile (last accessed date 30 October 2014).

Destatis (2013). Hintergrundtabelle zur Pressemitteilung vom 25.10.2013. Tabelle 0: Armutsgefährdungsschwelle in Deutschland. Statistisches Bundesamt. Available at: https://www.destatis.de (last accessed date 30 October 2014).

Eckenrode J, Smith EG, McCarthy ME, Dineen M (2014). Income inequality and child maltreatment in the United States. Pediatrics 133: 454-461.

Eiden RD, Homish GG, Colder CR, Schuetze P, Gray TR, Huestis MA (2013). Changes in smoking patterns during pregnancy. Subst Use Misuse 48: 513-522.

Emerson E, Einfeld S, Stancliffe RJ (2011). Predictors of the persistence of conduct difficulties in children with cognitive delay. J Child Psychol Psychiatry 52: 1184-1194.

Esser G, Blanz B, Geisel B, Laucht M (1989). Mannheim Parent Interview-Structured Interview for Child Psychiatric Disorders. Beltz: Weinheim, Germany.

Evans GW, Exner-Cortens D, Kim P, Bartholomew D (2013). Childhood poverty and blood pressure reactivity to and recovery from an acute stressor in late adolescence: the mediating role of family conflict. Psychosom Med 75: 691-700.

Eyles DW, Feron F, Cui X, Kesby JP, Harms LH, Ko P et al (2009). Developmental vitamin D deficiency causes abnormal brain development. Psychoneuroendocrinology 34(Suppl 1): S247-S257.

Fairchild G, Passamonti L, Hurford G, Hagan CC, von dem Hagen EA, van Goozen SH et al (2011). Brain structure abnormalities in early-onset and adolescent-onset conduct disorder. $A m \mathrm{~J}$ Psychiatry 168: 624-633.

Finger EC, Marsh AA, Blair KS, Reid ME, Sims C, Ng P et al (2011). Disrupted reinforcement signaling in the orbitofrontal cortex and caudate in youths with conduct disorder or oppositional defiant disorder and a high level of psychopathic traits. Am J Psychiatry 168: 152-162.

Flouri E, Mavroveli S, Panourgia C (2013). The role of general cognitive ability in moderating the relation of adverse life events to emotional and behavioural problems. $B r J$ Psychol 104: $130-139$.

Hamshere ML, Langley K, Martin J, Agha SS, Stergiakouli E, Anney RJ et al (2013). High loading of polygenic risk for ADHD in children with comorbid aggression. Am J Psychiatry 170: 909-916.

Hanson JL, Chandra A, Wolfe BL, Pollak SD (2011). Association between income and the hippocampus. PLoS One 6: e18712.

Hanson JL, Hair N, Shen DG, Shi F, Gilmore JH, Wolfe BL et al (2013). Family poverty affects the rate of human infant brain growth. PLoS One 8: e80954.

Hawkins JD, Catalano RF, Miller JY (1992). Risk and protective factors for alcohol and other drug problems in adolescence and early adulthood: implications for substance abuse prevention. Psychol Bull 112: 64-105.

Holz NE, Boecker R, Baumeister S, Hohm E, Zohsel K, Buchmann AF et al (2014). Effect of prenatal exposure to tobacco smoke on inhibitory control: neuroimaging results from a 25 -year prospective study. JAMA Psychiatry 71: 786-796.

Huebner T, Vloet TD, Marx I, Konrad K, Fink GR, Herpertz SC et al (2008). Morphometric brain abnormalities in boys with conduct disorder. J Am Acad Child Adolesc Psychiatry 47: 540-547.

Jednorog K, Altarelli I, Monzalvo K, Fluss J, Dubois J, Billard C et al (2012). The influence of socioeconomic status on children's brain structure. PLoS One 7: e42486.

Kaufman J, Birmaher B, Brent D, Rao U, Ryan N (1996). KiddieSads-Present and Lifetime Version (K-SADS_PL). Available at: http://www.psychiatry.pitt.edu/node/8233 (last accessed date 30 October 2014).

Kim P, Evans GW, Angstadt M, Ho SS, Sripada CS, Swain JE et al (2013). Effects of childhood poverty and chronic stress on emotion regulatory brain function in adulthood. Proc Natl Acad Sci USA 110: 18442-18447.

Kringelbach ML, Rolls ET (2004). The functional neuroanatomy of the human orbitofrontal cortex: evidence from neuroimaging and neuropsychology. Prog Neurobiol 72: 341-372.

Kunitz SJ, Levy JE, McCloskey J, Gabriel KR (1998). Alcohol dependence and domestic violence as sequelae of abuse and conduct disorder in childhood. Child Abuse Negl 22: 1079-1091.

Laucht M, Esser G, Baving L, Gerhold M, Hoesch I, Ihle W et al (2000). Behavioral sequelae of perinatal insults and early family adversity at 8 years of age. J Am Acad Child Adolesc Psychiatry 39: 1229-1237.

Laucht M, Esser G, Schmidt MH (2001). Differential development of infants at risk for psychopathology: the moderating role of early maternal responsivity. Dev Med Child Neurol 43: 292-300.

Lawson GM, Duda JT, Avants BB, Wu J, Farah MJ (2013). Associations between children's socioeconomic status and prefrontal cortical thickness. Dev Sci 16: 641-652.

Lelkes O, Gasior K (2011). Income Poverty in the EU-Situation in 2007 and Trends (based on EU-SILC 2005-2008). European Centre for Social Welfare Policy and Research. Available at: http:// www.euro.centre.org/data/1295444473_73292.pdf (last accessed date 30 October 2014).

Luby J, Belden A, Botteron K, Marrus N, Harms MP, Babb C et al (2013). The effects of poverty on childhood brain development: the mediating effect of caregiving and stressful life events. JAMA Pediatr 167: 1135-1142.

Lynch JW, Smith GD, Kaplan GA, House JS (2000). Income inequality and mortality: importance to health of individual income, psychosocial environment, or material conditions. BMJ 320: $1200-1204$.

Maier-Diewald W, Wittchen $\mathrm{H}-\mathrm{U}$, Hecht $\mathrm{H}$, Werner-Eilert $\mathrm{K}$ (1983). Munich Interview for the Assessment of Life Events and Conditions-Manual. Max Planck Institute of Psychiatry: Munich, Germany.

Maldjian JA, Laurienti PJ, Kraft RA, Burdette JH (2003). An automated method for neuroanatomic and cytoarchitectonic atlas-based interrogation of fMRI data sets. NeuroImage 19: 1233-1239.

Meyer-Lindenberg A, Tost H (2012). Neural mechanisms of social risk for psychiatric disorders. Nat Neurosci 15: 663-668.

Midouhas E, Yogaratnam A, Flouri E, Charman T (2013). Psychopathology trajectories of children with autism spectrum disorder: the role of family poverty and parenting. J Am Acad Child Adolesc Psychiatry 52: 1057-1065 e1051.

Moore CE, Radcliffe JD, Liu Y (2014). Vitamin D intakes of adults differ by income, gender and race/ethnicity in the USA, 2007 to 2010. Public Health Nutr 17: 756-763.

Noble KG, Houston SM, Kan E, Sowell ER (2012). Neural correlates of socioeconomic status in the developing human brain. Dev Sci 15: 516-527.

Nock MK, Kazdin AE, Hiripi E, Kessler RC (2006). Prevalence, subtypes, and correlates of DSM-IV conduct disorder in the National Comorbidity Survey Replication. Psychol Med 36: 699-710.

Potijk MR, Kerstjens JM, Bos AF, Reijneveld SA, de Winter AF (2013). Developmental delay in moderately preterm-born children with low socioeconomic status: risks multiply. J Pediatr 163: $1289-1295$. 
Rubia K (2011). 'Cool' inferior frontostriatal dysfunction in attention-deficit/hyperactivity disorder versus 'hot' ventromedial orbitofrontal-limbic dysfunction in conduct disorder: a review. Biol Psychiatry 69: e69-e87.

Rubia K, Smith AB, Halari R, Matsukura F, Mohammad M, Taylor E et al (2009). Disorder-specific dissociation of orbitofrontal dysfunction in boys with pure conduct disorder during reward and ventrolateral prefrontal dysfunction in boys with pure ADHD during sustained attention. Am J Psychiatry 166: 83-94.

Shelton KH, Collishaw S, Rice FJ, Harold GT, Thapar A (2011). Using a genetically informative design to examine the relationship between breastfeeding and childhood conduct problems. Eur Child Adolesc Psychiatry 20: 571-579.

Short K (2013). The Research Supplemental Poverty Measure: 2012. US Department of Commerce, Economics and Statistics Administration, US Census Bureau. Available at: http://www.census. gov/prod/2013pubs/p60-247.pdf.
Slotkin TA, Southard MC, Adam SJ, Cousins MM, Seidler FJ (2004). Alpha7 nicotinic acetylcholine receptors targeted by cholinergic developmental neurotoxicants: nicotine and chlorpyrifos. Brain Res Bull 64: 227-235.

Sripada RK, Swain JE, Evans GW, Welsh RC, Liberzon I (2014). Childhood poverty and stress reactivity are associated with aberrant functional connectivity in default mode network. Neuropsychopharmacology 39: 2244-2251.

US Census Bureau (2004). Current population survey: definitions and explanations. Available at: http://www.census.gov/cps/ about/cpsdef.html (last accessed date 30 October 2014).

Wittchen HU, Zaudig M, Fydrich T (1997). Structured Clinical Interview for DSM-IV Axis I and II-SCID. Hogrefe: Göttingen, Germany.

Yang Y, Raine A (2009). Prefrontal structural and functional brain imaging findings in antisocial, violent, and psychopathic individuals: a meta-analysis. Psychiatry Res 174: 81-88.

Supplementary Information accompanies the paper on the Neuropsychopharmacology website (http://www.nature.com/npp) 